\title{
ON A GENERALIZATION OF TEST IDEALS
}

\author{
NOBUO HARA AND SHUNSUKE TAKAGI
}

\begin{abstract}
The test ideal $\tau(R)$ of a ring $R$ of prime characteristic is an important object in the theory of tight closure. In this paper, we study a generalization of the test ideal, which is the ideal $\tau\left(\mathfrak{a}^{t}\right)$ associated to a given ideal $\mathfrak{a}$ with rational exponent $t \geq 0$. We first prove a key lemma of this paper (Lemma 2.1), which gives a characterization of the ideal $\tau\left(\mathfrak{a}^{t}\right)$. As applications of this key lemma, we generalize the preceding results on the behavior of the test ideal $\tau(R)$. Moreover, we prove an analogue of so-called Skoda's theorem, which is formulated algebraically via adjoint ideals by Lipman in his proof of the "modified Briançon-Skoda theorem."
\end{abstract}

\section{Introduction}

Let $R$ be a Noetherian commutative ring of characteristic $p>0$. The test ideal $\tau(R)$ of $R$, introduced by Hochster and Huneke [HH2], is defined to be the annihilator ideal of all tight closure relations in $R$ and plays an important role in the theory of tight closure. In [HY], the first-named author and Yoshida introduced a generalization of tight closure, which we call $\mathfrak{a}$-tight closure associated to a given ideal $\mathfrak{a}$, and defined the ideal $\tau(\mathfrak{a})$ to be the annihilator ideal of all $\mathfrak{a}$-tight closure relations in $R$. We can also consider $\mathfrak{a}^{t}$-tight closure and the ideal $\tau\left(\mathfrak{a}^{t}\right)$ with rational exponent $t \geq 0$ (or, rational coefficient in a sense), and even more, those with several rational exponents.

The ideals $\tau\left(\mathfrak{a}^{t}\right)$ have several nice properties similar to those of multiplier ideals $\mathcal{J}\left(\mathfrak{a}^{t}\right)$ defined via resolution of singularities in characteristic zero; see [La] for a systematic study of multiplier ideals. Among them, we have an analogue of Lipman's "modified Briançon-Skoda theorem" ([HY, Theorem 2.1], cf. [Li]) and the subadditivity theorem in regular local rings ([HY, Theorem 4.5], cf. [DEL]). It is notable that the above properties of

Received January 8, 2003.

2000 Mathematics Subject Classification: 13A35.

Both authors thank MSRI for the support and hospitality during their stay in the fall of 2002. The first-named author is partially supported by Grant-in-Aid for Scientific Research, Japan. 
the ideals $\tau\left(\mathfrak{a}^{t}\right)$ are proved quite algebraically via characteristic $p$ methods. On the other hand, we can prove that the multiplier ideal $\mathcal{J}\left(\mathfrak{a}^{t}\right)$ in a normal $\mathbb{Q}$-Gorenstein ring of characteristic zero coincides, after reduction to characteristic $p \gg 0$, with the ideal $\tau\left(\mathfrak{a}^{t}\right)$; see [HY] and also [Ta].

In this paper, we study further properties of $\mathfrak{a}^{t}$-tight closure and the ideal $\tau\left(\mathfrak{a}^{t}\right)$ in characteristic $p>0$, improve results obtained in $[\mathrm{HY}]$, and give some applications. To do this, we first prove a key lemma of this paper (Lemma 2.1), which gives a characterization of (elements of) the ideal $\tau\left(\mathfrak{a}^{t}\right)$. Precisely speaking, Lemma 2.1 characterizes the ideal $\tilde{\tau}\left(\mathfrak{a}^{t}\right)$ given in Definition 1.4, which is contained in the ideal $\tau\left(\mathfrak{a}^{t}\right)$ and expected to coincide with $\tau\left(\mathfrak{a}^{t}\right)$. The identification of the ideals $\tau\left(\mathfrak{a}^{t}\right)$ and $\tilde{\tau}\left(\mathfrak{a}^{t}\right)$ holds true in some reasonable situations, e.g., in normal $\mathbb{Q}$-Gorenstein rings; see $[\mathrm{AM}],[\mathrm{HY}]$ and $[\mathrm{LS} 2]$.

Although the description of Lemma 2.1 is somewhat complicated, it turns out to be very useful in studying various properties of the ideals $\tau\left(\mathfrak{a}^{t}\right)$. As applications of this key lemma, we answer a question raised in [HY] (Corollary 2.3) and consider the relationship of test elements and $\mathfrak{a}^{t}$ test elements (Corollary 2.4). We also apply Lemma 2.1 to the study of the behavior of the ideals $\tau\left(\mathfrak{a}^{t}\right)$ under localization (Proposition 3.1), completion (Proposition 3.2) and finite morphisms which are étale in codimension one (Theorem 3.3). These results generalize the preceding results [LS2] and [BSm] on the behavior of the test ideal $\tau(R)$, and we hope that the proofs become simpler with the use of Lemma 2.1 .

Other ingredients of this paper are Theorems 4.1 and 4.2, which assert that if $\mathfrak{a}$ is an ideal with a reduction generated by $l$ elements, then $\tau\left(\mathfrak{a}^{l}\right)=$ $\tau\left(\mathfrak{a}^{l-1}\right) \mathfrak{a}$. This is an analogue of so-called Skoda's theorem [La], which is formulated algebraically via adjoint ideals (or multiplier ideals) by Lipman [Li] in his proof of the "modified Briançon-Skoda theorem." Theorem 4.1 is a refinement of [HY, Theorem 2.1] and is proved by an easy observation on the relationship of regular powers and Frobenius powers of ideals (cf. $[\mathrm{AH}])$. Theorem 4.2 is based on the same idea, but is proved under a slightly different assumption with the aid of Lemma 2.1. We can find an advantage of the ideal $\tau(\mathfrak{a})$ in the simplicity of the proofs of Theorems 4.1 and 4.2 , because the proof of Skoda's theorem for multiplier ideals needs a deep vanishing theorem which is proved only in characteristic zero [La], [Li]. 


\section{§1. Preliminaries}

In this paper, all rings are excellent reduced commutative rings with unity. For a ring $R$, we denote by $R^{\circ}$ the set of elements of $R$ which are not in any minimal prime ideal. Let $R$ be a ring of prime characteristic $p>0$ and $F: R \rightarrow R$ the Frobenius map which sends $x \in R$ to $x^{p} \in R$. The ring $R$ viewed as an $R$-module via the $e$-times iterated Frobenius map $F^{e}: R \rightarrow R$ is denoted by ${ }^{e} R$. Since $R$ is assumed to be reduced, we can identify $F: R \rightarrow{ }^{e} R$ with the natural inclusion map $R \hookrightarrow R^{1 / p^{e}}$. We say that $R$ is $F$-finite if ${ }^{1} R$ (or $R^{1 / p}$ ) is a finitely generated $R$-module.

Let $R$ be a ring of characteristic $p>0$ and let $M$ be an $R$-module. For each $e \in \mathbb{N}$, we denote $\mathbb{F}^{e}(M)=\mathbb{F}_{R}^{e}(M):={ }^{e} R \otimes_{R} M$ and regard it as an $R$-module by the action of $R={ }^{e} R$ from the left. Then we have the induced $e$-times iterated Frobenius map $F^{e}: M \rightarrow \mathbb{F}^{e}(M)$. The image of $z \in M$ via this map is denoted by $z^{q}:=F^{e}(z) \in \mathbb{F}^{e}(M)$. For an $R$-submodule $N$ of $M$, we denote by $N_{M}^{[q]}$ the image of the induced map $\mathbb{F}^{e}(N) \rightarrow \mathbb{F}^{e}(M)$.

Now we recall the definition of $\mathfrak{a}^{t}$-tight closure. See $[\mathrm{HY}]$ for details.

Definition 1.1. Let $\mathfrak{a}$ be an ideal of a ring $R$ of characteristic $p>0$ such that $\mathfrak{a} \cap R^{\circ} \neq \emptyset$, and let $N \subseteq M$ be $R$-modules. Given a rational number $t \geq 0$, the $\mathfrak{a}^{t}$-tight closure $N_{M}^{* \mathfrak{a}^{t}}$ of $N$ in $M$ is defined to be the submodule of $M$ consisting of all elements $z \in M$ for which there exists $c \in R^{\circ}$ such that

$$
c z^{q} \mathfrak{a}^{\lceil t q\rceil} \subseteq N_{M}^{[q]}
$$

for all large $q=p^{e}$, where $\lceil t q\rceil$ is the least integer which is greater than or equal to $t q$. The $\mathfrak{a}^{t}$-tight closure $I^{* \mathfrak{a}^{t}}$ of an ideal $I \subseteq R$ is just defined by $I^{* \mathfrak{a}^{t}}=I_{R}^{* \mathrm{a}^{t}}$.

Remark 1.2. The rational exponent $t$ for $\mathfrak{a}^{t}$-tight closure in Definition 1.1 is just a formal notation, but it is compatible with "real" powers of the ideal. Namely, if $\mathfrak{b}=\mathfrak{a}^{n}$ for $n \in \mathbb{N}$, then $\mathfrak{a}^{t}$-tight closure is the same as $\mathfrak{b}^{t / n}$ tight closure. This allows us to extend the definition to several rational exponents: Given ideals $\mathfrak{a}_{1}, \ldots, \mathfrak{a}_{r} \subseteq R$ with $\mathfrak{a}_{i} \cap R^{\circ} \neq \emptyset$ and rational numbers $t_{1}, \ldots, t_{r} \geq 0$, if $t_{i}=t n_{i}$ for nonnegative $t \in \mathbb{Q}$ and $n_{i} \in \mathbb{N}$ with $i=1, \ldots, r$, we can define $\mathfrak{a}_{1}^{t_{1}} \cdots \mathfrak{a}_{r}^{t_{r}}$-tight closure to be $\left(\mathfrak{a}_{1}^{n_{1}} \cdots \mathfrak{a}_{r}^{n_{r}}\right)^{t}$-tight closure. If $N$ is a submodule of an $R$-module $M$, then an element $z \in M$ is in the $\mathfrak{a}_{1}^{t_{1}} \cdots \mathfrak{a}_{r}^{t_{r}}$-tight closure $N_{M}^{* \mathfrak{a}_{1}^{t_{1}} \cdots \mathfrak{a}_{r}^{t_{r}}}$ of $N$ in $M$ if and only if there exists $c \in R^{\circ}$ such that $c z^{q} \mathfrak{a}_{1}^{\left\lceil t_{1} q\right\rceil} \cdots \mathfrak{a}_{r}^{\left[t_{r} q\right\rceil} \subseteq N_{M}^{[q]}$ for all $q=p^{e} \gg 0$. 
Since $N_{M}^{* \mathfrak{a}^{t}} / N \cong 0_{M / N}^{* \mathfrak{a}^{t}}$ for $R$-modules $N \subseteq M$ ([HY, Proposition 1.3 (1)]), the case where $N=0$ is essential. Using the $\mathfrak{a}^{t}$-tight closure of the zero submodule, we can define two ideals $\tau\left(\mathfrak{a}^{t}\right)$ and $\tilde{\tau}\left(\mathfrak{a}^{t}\right)$.

Proposition-Definition 1.3. ([HY, Definition-Theorem 6.5]) Let $R$ be an excellent reduced ring of characteristic $p>0, \mathfrak{a} \subseteq R$ an ideal such that $\mathfrak{a} \cap R^{\circ} \neq \emptyset$ and $t \geq 0$ a rational number. Let $E=\bigoplus_{\mathfrak{m}} E_{R}(R / \mathfrak{m})$ be the direct sum, taken over all maximal ideals $\mathfrak{m}$ of $R$, of the injective envelopes of the residue fields $R / \mathfrak{m}$. Then the following ideals are equal to each other and we denote it by $\tau\left(\mathfrak{a}^{t}\right)$.

(i) $\bigcap_{M} \operatorname{Ann}_{R}\left(0_{M}^{* \mathfrak{a}^{t}}\right)$, where $M$ runs through all finitely generated $R$-modules.

(ii) $\bigcap_{M \subseteq E} \operatorname{Ann}_{R}\left(0_{M}^{* \mathfrak{a}^{t}}\right)$, where $M$ runs through all finitely generated submodules of $E$.

(iii) $\bigcap_{J \subseteq R}\left(J: J^{* \mathfrak{a}^{t}}\right)$, where $J$ runs through all ideals of $R$.

The description (ii) of $\tau\left(\mathfrak{a}^{t}\right)$ in Proposition-Definition 1.3 means that $\tau\left(\mathfrak{a}^{t}\right)$ is the annihilator ideal of the "finitistic $\mathfrak{a}^{t}$-tight closure" of zero in $E$, that is, the union of $0_{M}^{* \mathfrak{a}^{t}}$ in $E$ taken over all finitely generated submodules $M$ of $E$. It would be natural to consider the $\mathfrak{a}^{t}$-tight closure of zero in $E$ instead of the finitistic tight closure.

Definition 1.4. Let $E=\bigoplus_{\mathfrak{m}} E_{R}(R / \mathfrak{m})$ be as in Proposition-Definition 1.3. Then we define the ideal $\tilde{\tau}\left(\mathfrak{a}^{t}\right)$ by

$$
\tilde{\tau}\left(\mathfrak{a}^{t}\right)=\operatorname{Ann}_{R}\left(0_{E}^{* \mathfrak{a}^{t}}\right)
$$

We have $\tilde{\tau}\left(\mathfrak{a}^{t}\right) \subseteq \tau\left(\mathfrak{a}^{t}\right)$ in general, since $0_{M}^{* \mathfrak{a}^{t}} \subseteq 0_{E}^{* \mathfrak{a}^{t}}$ for all (finitely generated) submodules $M$ of $E$. We do not know any example in which the $\mathfrak{a}^{t}$-tight closure of zero and the finitistic $\mathfrak{a}^{t}$-tight closure of zero disagree in $E$, and it seems reasonable to assume the following condition.

(1.4.1) We say that condition (*) is satisfied for $\mathfrak{a}^{t}$ if $\tau\left(\mathfrak{a}^{t}\right)=\tilde{\tau}\left(\mathfrak{a}^{t}\right)$. 
Condition $(*)$ is satisfied in many situations. For example, if $R$ is a graded ring, then condition $(*)$ is satisfied for the unit ideal $R$ (see [LS1]), and if $R$ is an excellent $\mathbb{Q}$-Gorenstein normal local ring, then condition (*) is satisfied for every rational number $t \geq 0$ and every ideal $\mathfrak{a} \subseteq R$ such that $\mathfrak{a} \cap R^{\circ} \neq \emptyset$ ([HY, Theorem 1.13]; see also [AM]).

In most part of this paper, we will assume condition $(*)$ to deduce various properties of the ideal $\tau\left(\mathfrak{a}^{t}\right)$, because what we actually do is to prove those properties for the ideal $\tilde{\tau}\left(\mathfrak{a}^{t}\right)$, which are translated into those for $\tau\left(\mathfrak{a}^{t}\right)$ under condition $(*)$. So, we remark that most of our results do hold true for $\tilde{\tau}\left(\mathfrak{a}^{t}\right)$ without assuming condition $(*)$.

Finally we recall the notion of an $\mathfrak{a}^{t}$-test element, which is quite useful to handle the $\mathfrak{a}^{t}$-tight closure operation. See [HY, Theorems 1.7 and 6.4] for the existence of $\mathfrak{a}^{t}$-test elements.

Definition 1.5. Let $\mathfrak{a}$ be an ideal of a ring $R$ of characteristic $p>0$ such that $\mathfrak{a} \cap R^{\circ} \neq \emptyset$, and let $t \geq 0$ be a rational number. An element $d \in R^{\circ}$ is called an $\mathfrak{a}^{t}$-test element if for every finitely generated $R$-module $M$ and every element $z \in M$, the following holds: $z \in 0_{M}^{* \mathfrak{a}^{t}}$ if and only if $d z^{q} \mathfrak{a}^{\lceil t q\rceil}=0$ for all powers $q=p^{e}$ of $p$.

Remark 1.6. In the case where $\mathfrak{a}=R$ is the unit ideal, the ideal $\tau(\mathfrak{a})=$ $\tau(R)$ is called the test ideal of $R$ and an $R$-test element is nothing but a test element as defined in [HH2]. In this case, $\tau(R) \cap R^{\circ}$ is exactly equal to the set of test elements. However, $\tau\left(\mathfrak{a}^{t}\right) \cap R^{\circ}$ is not equal to the set of $\mathfrak{a}^{t}$-test elements in general. The relationship between the ideal $\tau\left(\mathfrak{a}^{t}\right)$ and $\mathfrak{a}^{t}$-test elements is not a priori clear, but we will see later in Corollary 2.4 that an element of $\tau\left(\mathfrak{a}^{t}\right) \cap R^{\circ}$ is always an $\mathfrak{a}^{t}$-test element.

The reader is referred to [HY] for basic properties of $\mathfrak{a}^{t}$-tight closure and the ideal $\tau\left(\mathfrak{a}^{t}\right)$. Among them, see especially Propositions 1.3 and 1.11 and Theorem 1.7, together with Section 6, of [HY].

All results in this paper are proved in characteristic $p>0$. But it may help better understanding of the results to keep in mind the correspondence of the ideals $\tau\left(\mathfrak{a}^{t}\right)$ and the multiplier ideals defined via resolution of singularities in characteristic zero ([HY]). Namely, given a rational number $t \geq 0$ and an ideal $\mathfrak{a}$ of a normal $\mathbb{Q}$-Gorenstein ring essentially of finite type over a field of characteristic zero, the multiplier ideal $\mathcal{J}\left(\mathfrak{a}^{t}\right)$ coincides, after reduction to characteristic $p \gg 0$, with the ideal $\tau\left(a^{t}\right)$. 


\section{§2. A characterization of the ideal $\tau\left(\mathfrak{a}^{t}\right)$}

We first show a key lemma of this paper, which gives a characterization of the ideal $\tilde{\tau}\left(\mathfrak{a}^{t}\right)$ and reveals the relationship between the ideal $\tau\left(\mathfrak{a}^{t}\right)$ and $\mathfrak{a}^{t}$-test elements under condition $(*)$. Its essential idea is found in [Ta, Theorem 3.13].

Lemma 2.1. Let $(R, \mathfrak{m})$ be an $F$-finite local ring of characteristic $p>0$, $\mathfrak{a} \subseteq R$ an ideal such that $\mathfrak{a} \cap R^{\circ} \neq \emptyset$ and $t \geq 0$ a rational number. Fix a system of generators $x_{1}^{(e)}, \ldots, x_{r_{e}}^{(e)}$ of $\mathfrak{a}^{\lceil t q\rceil}$ for each $q=p^{e}$. Then for any element $c \in R$, the following four conditions are equivalent to each other.

(i) $c \in \tilde{\tau}\left(\mathfrak{a}^{t}\right)$.

(ii) For any element $d \in R^{\circ}$ and any integer $e_{0} \geq 0$, there exist an integer $e_{1} \geq e_{0}$ and $R$-homomorphisms $\phi_{i}^{(e)} \in \operatorname{Hom}_{R}\left(R^{1 / p^{e}}, R\right)$ for $e_{0} \leq e \leq$ $e_{1}$ and $1 \leq i \leq r_{e}$ such that

$$
c=\sum_{e=e_{0}}^{e_{1}} \sum_{i=1}^{r_{e}} \phi_{i}^{(e)}\left(\left(d x_{i}^{(e)}\right)^{1 / p^{e}}\right) .
$$

(iii) For an $\mathfrak{a}^{t}$-test element $d \in R^{\circ}$, there exist a positive integer $e_{1}$ and $R$-linear maps $\phi_{i}^{(e)} \in \operatorname{Hom}_{R}\left(R^{1 / p^{e}}, R\right)$ for $0 \leq e \leq e_{1}$ and $1 \leq i \leq r_{e}$ such that

$$
c=\sum_{e=0}^{e_{1}} \sum_{i=1}^{r_{e}} \phi_{i}^{(e)}\left(\left(d x_{i}^{(e)}\right)^{1 / p^{e}}\right) .
$$

(iii) $^{\prime}$ For an $\mathfrak{a}^{t}$-test element $d \in R^{\circ}$, there exist a positive integer $e_{1}$ and $R$-linear maps $\phi_{i}^{(e)} \in \operatorname{Hom}_{R}\left(R^{1 / p^{e}}, R\right)$ for $0 \leq e \leq e_{1}$ and $1 \leq i \leq r_{e}$ such that

$$
c \in \sum_{e=0}^{e_{1}} \sum_{i=1}^{r_{e}} \phi_{i}^{(e)}\left(\left(d \mathfrak{a}^{\left\lceil t p^{e}\right\rceil}\right)^{1 / p^{e}}\right) .
$$

Proof. We prove the equivalence of conditions (i) and (ii). Let $F^{e}: E_{R}$ $\rightarrow{ }^{e} R \otimes_{R} E_{R}$ be the $e$-times iterated Frobenius map induced on the injective envelope $E_{R}:=E_{R}(R / \mathfrak{m})$ of the residue field $R / \mathfrak{m}$. For an element $d \in R^{\circ}$ and an integer $e \geq 0$, we write

$$
d \mathbf{x}^{(e)} F^{e}:={ }^{t}\left(d x_{1}^{(e)} F^{e}, \ldots, d x_{r_{e}}^{(e)} F^{e}\right): E_{R} \rightarrow\left({ }^{e} R \otimes_{R} E_{R}\right)^{\oplus r_{e}} .
$$


By definition and condition $(*), c \in \tau\left(\mathfrak{a}^{t}\right)$ if and only if $c \cdot \bigcap_{e \geq e_{0}} \operatorname{Ker}\left(d \mathbf{x}^{(e)} F^{e}\right)$ $=0$ holds for every element $d \in R^{\circ}$ and every integer $e_{0} \geq 0$. Since $E_{R}$ is an Artinian $R$-module, there exists an integer $e_{1} \geq e_{0}$ (depending on $d \in R^{\circ}$ and $\left.e_{0} \geq 0\right)$ such that

$$
\bigcap_{e \geq e_{0}} \operatorname{Ker}\left(d \mathbf{x}^{(e)} F^{e}\right)=\bigcap_{e=e_{0}}^{e_{1}} \operatorname{Ker}\left(d \mathbf{x}^{(e)} F^{e}\right) .
$$

Therefore denoting

$$
\Phi=\Phi_{d}^{\left(e_{0}, e_{1}\right)}:={ }^{t}\left(d \mathbf{x}^{\left(e_{0}\right)} F^{e_{0}}, \ldots, d \mathbf{x}^{\left(e_{1}\right)} F^{e_{1}}\right): E_{R} \rightarrow \bigoplus_{e=e_{0}}^{e_{1}}\left({ }^{e} R \otimes_{R} E_{R}\right)^{\oplus r_{e}},
$$

we see that $c \in \tau\left(\mathfrak{a}^{t}\right)$ if and only if for every $d \in R^{\circ}$ and $e_{0} \geq 0$, there exists $e_{1} \geq e_{0}$ such that $c \cdot \operatorname{Ker}\left(\Phi_{d}^{\left(e_{0}, e_{1}\right)}\right)=0$.

Since $R$ is F-finite, the map $\Phi=\Phi_{d}^{\left(e_{0}, e_{1}\right)}: E_{R} \rightarrow \bigoplus_{e=e_{0}}^{e_{1}}\left({ }^{e} R \otimes_{R} E_{R}\right)^{\oplus r_{e}}$ is the Matlis dual of the map

$$
\Psi=\left(\psi^{\left(e_{0}\right)}, \ldots, \psi^{\left(e_{1}\right)}\right): \bigoplus_{e=e_{0}}^{e_{1}} \operatorname{Hom}_{R}\left(R^{1 / p^{e}}, R\right)^{\oplus r_{e}} \rightarrow R,
$$

where the map $\psi^{(e)}: \operatorname{Hom}_{R}\left(R^{1 / p^{e}}, R\right)^{\oplus r_{e}} \rightarrow \operatorname{Hom}_{R}(R, R)=R$ is induced by the $R$-linear map $R \rightarrow\left(R^{1 / p^{e}}\right)^{\oplus r_{e}}$ sending 1 to $\left(\left(d x_{1}^{(e)}\right)^{1 / p^{e}}, \ldots,\left(d x_{r_{e}}^{(e)}\right)^{1 / p^{e}}\right)$. It then follows that $c \cdot \operatorname{Ker}(\Phi)=0$ if and only if $c \in \operatorname{Im}(\Psi)$. By the definition of $\Psi$, this is equivalent to saying that there exist $R$-linear maps $\phi_{i}^{(e)} \in \operatorname{Hom}_{R}\left(R^{1 / p^{e}}, R\right)$ for $e_{0} \leq e \leq e_{1}$ and $1 \leq i \leq r_{e}$ such that

$$
c=\sum_{e=e_{0}}^{e_{1}} \sum_{i=1}^{r_{e}} \phi_{i}^{(e)}\left(\left(d x_{i}^{(e)}\right)^{1 / p^{e}}\right) .
$$

The equivalence of conditions (i) and (iii) (resp. (iii) $)^{\prime}$ ) is obtained in the same way.

Remark 2.2. An advantage of Lemma 2.1 is that it is applicable even in the absense of the completeness of $R$. For example, compare the hypotheses in Theorem 4.1 and Theorem 4.2.

As immediate consequences of Lemma 2.1, we have the following corollaries. First Lemma 2.1 gives an affirmative answer to a question raised in [HY, Discussion 5.18]. 
Corollary 2.3. Let $(R, \mathfrak{m})$ be an F-finite local ring of characteristic $p>0$, and suppose that condition $(*)$ is satisfied for the maximal ideal $\mathfrak{m}$. Then $\tau(\mathfrak{m})=R$ if and only if for every $\mathfrak{m}$-primary ideal $\mathfrak{a} \subset R$, we have a strict containment $\tau(\mathfrak{a}) \supsetneq \mathfrak{a}$. In particular, if $R$ is a regular local ring with $\operatorname{dim} R \geq 2$, then $\tau(\mathfrak{a}) \supsetneq \mathfrak{a}$ for every $\mathfrak{m}$-primary ideal $\mathfrak{a} \subset R$.

Proof. Suppose that $\tau(\mathfrak{m})=R$. We will show that $\tau(\mathfrak{a}) \supseteq(\mathfrak{a}: \mathfrak{m})$ for every ideal $\mathfrak{a} \subseteq R$ such that $\mathfrak{a} \cap R^{\circ} \neq \emptyset$. By Lemma 2.1 , for any element $d \in$ $R^{\circ}$ and any integer $e_{0} \geq 0$, there exist an integer $e \geq e_{0}$, an element $x \in \mathfrak{m}^{p^{e}}$ and an $R$-module homomorphism $\phi: R^{1 / p^{e}} \rightarrow R$ such that $\phi\left((d x)^{1 / p^{e}}\right)=1$. Now fix any element $y \in(\mathfrak{a}: \mathfrak{m})$. Since $\phi\left(\left(d x y^{p^{e}}\right)^{1 / p^{e}}\right)=\phi\left((d x)^{1 / p^{e}}\right) y=y$ and $x y^{p^{e}} \in \mathfrak{a}^{p^{e}}$, we have $y \in \tau(\mathfrak{a})$ by using Lemma 2.1 again. The converse implication is trivial. The latter assertion follows from [HY, Theorem 2.15].

Corollary 2.4. Let $(R, \mathfrak{m})$ be an F-finite local ring of characteristic $p>0$. If condition $(*)$ is satisfied for the unit ideal $R$, then a test element of $R$ is an $\mathfrak{a}^{t}$-test element for all ideals $\mathfrak{a} \subseteq R$ such that $\mathfrak{a} \cap R^{\circ} \neq \emptyset$ and all rational numbers $t \geq 0$.

Proof. Let $c$ be a test element of $R$, that is, an element of $\tau(R) \cap R^{\circ}$. Given any ideals $\mathfrak{a}, J$ of $R$, any rational number $t \geq 0$, any element $z \in J^{* \mathfrak{a}^{t}}$ and any power $q$ of $p$, it is enough to show that $c z^{q} \mathfrak{a}^{\lceil t q\rceil} \subseteq J^{[q]}$. Since $z \in J^{* \mathfrak{a}^{t}}$, there exist $d \in R^{\circ}$ and $e_{0} \in \mathbb{N}$ such that $d z^{Q} \mathfrak{a}^{\lceil t \bar{Q}\rceil} \subseteq J^{[Q]}$ for every power $Q \geq p^{e_{0}}$ of $p$. Then by Lemma 2.1 , there exist $e_{1} \in \mathbb{N}$ and $\phi_{e} \in \operatorname{Hom}_{R}\left(R^{1 / p^{e}}, R\right)$ for $e_{0} \leq e \leq e_{1}$ such that $c=\sum_{e=e_{0}}^{e_{1}} \phi_{e}\left(d^{1 / p^{e}}\right)$. Since $d z^{q p^{e}}\left(\mathfrak{a}^{\lceil t q\rceil}\right)^{\left[p^{e}\right]} \subseteq d z^{q p^{e}} \mathfrak{a}^{\left\lceil t q p^{e}\right\rceil} \subseteq J^{\left[q p^{e}\right]}$ for every $e_{0} \leq e \leq e_{1}$, we have

$$
d^{1 / p^{e}} z^{q} \mathfrak{a}^{\lceil t q\rceil} R^{1 / p^{e}} \subseteq J^{[q]} R^{1 / p^{e}} .
$$

Applying $\phi_{e}$ and summing up, we obtain

$$
c z^{q} \mathfrak{a}^{\lceil t q\rceil}=\sum_{e=e_{0}}^{e_{1}} \phi_{e}\left(d^{1 / p^{e}}\right) z^{q} \mathfrak{a}^{\lceil t q\rceil} \subseteq \sum_{e=e_{0}}^{e_{1}} \phi_{e}\left(J^{[q]} R^{1 / p^{e}}\right) \subseteq J^{[q]},
$$

as required.

Remark 2.5. Since $\tilde{\tau}\left(\mathfrak{a}^{t}\right) \subseteq \tilde{\tau}(R)$, we see from Corollary 2.4 that any element of $\tau\left(\mathfrak{a}^{t}\right) \cap R^{\circ}$ is an $\mathfrak{a}^{t}$-test element as long as condition (*) is satisfied for $\mathfrak{a}^{t}$. Also, Corollary 2.4 is considered a refinement of [HY, Theorem 1.7], which asserts that, if the localized ring $R_{c}$ at an element $c \in R^{\circ}$ is strongly 
F-regular, then some power $c^{n}$ of $c$ is an $\mathfrak{a}^{t}$-test element for all ideals $\mathfrak{a} \subseteq R$ such that $\mathfrak{a} \cap R^{\circ} \neq \emptyset$ and all rational numbers $t \geq 0$. Indeed, if $R_{c}$ is strongly F-regular, then some power $c^{n}$ of $c$ is a test element (precisely speaking, an element of $\tilde{\tau}(R) \cap R^{\circ}$ ) by [HH1] (see also [HH3]), so that $c^{n}$ is an $\mathfrak{a}^{t}$-test element for all $\mathfrak{a}$ and $t$ by Corollary 2.4.

\section{§3. Behavior of $\tau(\mathfrak{a})$ under localization, completion and finite homomorphisms}

Proposition 3.1. (cf. [LS2]) Let $(R, \mathfrak{m})$ be an F-finite local ring of characteristic $p>0, \mathfrak{a} \subseteq R$ an ideal such that $\mathfrak{a} \cap R^{\circ} \neq \emptyset$ and $t \geq 0$ a rational number. Let $W$ be a multiplicatively closed subset of $R$ and suppose that condition $(*)$ is satisfied for $\mathfrak{a}^{t}$ and $\left(\mathfrak{a} R_{W}\right)^{t}$. Then

$$
\tau\left(\left(\mathfrak{a} R_{W}\right)^{t}\right)=\tau\left(\mathfrak{a}^{t}\right) R_{W} .
$$

Proof. Fix a system of generators $x_{1}^{(e)}, \ldots, x_{r_{e}}^{(e)}$ of $\mathfrak{a}^{\lceil t q\rceil}$ for each $q=p^{e}$. If an element $c \in R_{W}$ is contained in $\tau\left(\left(\mathfrak{a} R_{W}\right)^{t}\right)$, then by Lemma 2.1, for any element $d \in R^{\circ}$ and any nonnegative integer $e_{0}$, there exist an integer $e_{1} \geq e_{0}$ and $R_{W}$-homomorphisms $\phi_{i}^{(e)} \in \operatorname{Hom}_{R_{W}}\left(R_{W}^{1 / p^{e}}, R_{W}\right)$ for $e_{0} \leq e \leq e_{1}$ and $1 \leq i \leq r_{e}$ such that

$$
c=\sum_{e=e_{0}}^{e_{1}} \sum_{i=1}^{r_{e}} \phi_{i}^{(e)}\left(\left(d x_{i}^{(e)}\right)^{1 / p^{e}}\right) .
$$

Since $R$ is F-finite, there exists an element $y \in W$ such that we can regard $y \phi_{i}^{(e)}$ as an element of $\operatorname{Hom}_{R}\left(R^{1 / p^{e}}, R\right)$ for all $e_{0} \leq e \leq e_{1}$ and $1 \leq i \leq$ $r_{e}$. Therefore, thanks to Lemma 2.1 again, we have $c y \in \tau\left(\mathfrak{a}^{t}\right)$, that is, $c \in \tau\left(\mathfrak{a}^{t}\right) R_{W}$. The converse argument just reverses this. The proposition is proved.

Proposition 3.2. (cf. [LS2]) Let $(R, \mathfrak{m})$ be an F-finite local ring of characteristic $p>0, \mathfrak{a} \subseteq R$ an ideal such that $\mathfrak{a} \cap R^{\circ} \neq \emptyset$ and $t \geq 0$ a rational number. Let $\widehat{R}$ denote the $\mathfrak{m}$-adic completion of $R$ and suppose that condition $(*)$ is satisfied for $\mathfrak{a}^{t}$ and $(a \widehat{R})^{t}$. Then

$$
\tau\left((\mathfrak{a} \widehat{R})^{t}\right)=\tau\left(\mathfrak{a}^{t}\right) \widehat{R}
$$

Proof. Fix a system of generators $x_{1}^{(e)}, \ldots, x_{r_{e}}^{(e)}$ of $\mathfrak{a}^{\lceil t q\rceil}$ for each $q=p^{e}$. Since $R$ is F-finite, we can take an element $d \in R^{\circ}$ which is an $\mathfrak{a}^{t}$ - and 
$(\mathfrak{a} \widehat{R})^{t}$-test element by [HY, Theorem 6.4]. If an element $c \in \widehat{R}$ is contained in $\tau\left((\mathfrak{a} \widehat{R})^{t}\right)$, then by Lemma 2.1 , there exist an integer $e_{1}>0$ and $\widehat{R}$ homomorphisms $\phi_{i}^{(e)} \in \operatorname{Hom}_{\widehat{R}}\left(\widehat{R}^{1 / p^{e}}, \widehat{R}\right)$ for $0 \leq e \leq e_{1}$ and $1 \leq i \leq r_{e}$ such that

$$
c=\sum_{e=0}^{e_{1}} \sum_{i=1}^{r_{e}} \phi_{i}^{(e)}\left(\left(d x_{i}^{(e)}\right)^{1 / p^{e}}\right) .
$$

Since $R$ is F-finite, $\operatorname{Hom}_{\widehat{R}}\left(\widehat{R}^{1 / p^{e}}, \widehat{R}\right) \cong \widehat{R} \otimes_{R} \operatorname{Hom}_{R}\left(R^{1 / p^{e}}, R\right)$, so that there exist $y_{i, 1}^{(e)}, \ldots, y_{i, s_{e, i}}^{(e)} \in \widehat{R}$ and $\psi_{i, 1}^{(e)}, \ldots, \psi_{i, s_{e, i}}^{(e)} \in \operatorname{Hom}_{R}\left(R^{1 / p^{e}}, R\right)$ with $\phi_{i}^{(e)}=$ $\sum_{j=1}^{s_{e, i}} y_{i, j}^{(e)} \otimes \psi_{i, j}^{(e)}$ for all $0 \leq e \leq e_{1}$ and $1 \leq i \leq r_{e}$. Then

$$
c=\sum_{e=0}^{e_{1}} \sum_{i=1}^{r_{e}} \sum_{j=1}^{s_{e, i}} y_{i, j}^{(e)} \psi_{i, j}^{(e)}\left(\left(d x_{i}^{(e)}\right)^{1 / p^{e}}\right)
$$

Therefore, thanks to Lemma 2.1 again, we have $c \in \tau\left(\mathfrak{a}^{t}\right) \widehat{R}$. The converse argument just reverses this. The proposition is proved.

Our characterization is also applicable to the following situation; see also $[\mathrm{BSm}]$.

TheOREm 3.3. Let $(R, \mathfrak{m}) \hookrightarrow(S, \mathfrak{n})$ be a pure finite local homomorphism of F-finite normal local rings of characteristic $p>0$ which is étale in codimension one. Let $\mathfrak{a}$ be a nonzero ideal of $R$ and let $t$ be a nonnegative rational number. Assume that condition $(*)$ is satisfied for $\mathfrak{a}^{t}$ and $(\mathfrak{a} S)^{t}$. Then

$$
\tau\left((\mathfrak{a} S)^{t}\right) \cap R=\tau\left(\mathfrak{a}^{t}\right) .
$$

Moreover if $R \hookrightarrow S$ is flat, then

$$
\tau\left((\mathfrak{a} S)^{t}\right)=\tau\left(\mathfrak{a}^{t}\right) S
$$

Proof. Since $R \hookrightarrow S$ is pure, we have $\tau\left((\mathfrak{a} S)^{t}\right) \cap R \subseteq \tau\left(\mathfrak{a}^{t}\right)$ by $[\mathrm{HY}$, Proposition 1.12]. We will prove the reverse inclusion. Fix a system of generators $x_{1}^{(e)}, \ldots, x_{r_{e}}^{(e)}$ of $\mathfrak{a}^{\lceil t q\rceil}$ for every $q=p^{e}$. Take an element $d \in R^{\circ}$ which is not only an $\mathfrak{a}^{t}$-test element but also an $(\mathfrak{a} S)^{t}$-test element (cf. Remark 2.5, [BSm, Remark 6.5]). If an element $c$ belongs to $\tau\left(\mathfrak{a}^{t}\right)$, then by Lemma 2.1, there exist an integer $e_{1}>0$ and $R$-linear maps $\phi_{i}^{(e)} \in$ $\operatorname{Hom}_{R}\left(R^{1 / p^{e}}, R\right)$ for $0 \leq e \leq e_{1}$ and $1 \leq i \leq r_{e}$ such that

$$
c=\sum_{e=0}^{e_{1}} \sum_{i=1}^{r_{e}} \phi_{i}^{(e)}\left(\left(d x_{i}^{(e)}\right)^{1 / p^{e}}\right) .
$$


Let $c_{e, i}=\phi_{i}^{(e)}\left(\left(d x_{i}^{(e)}\right)^{1 / p^{e}}\right)$ and let $E_{S}=E_{S}(S / \mathfrak{n})$ be the injective envelope of the residue field of $S$. Since $R \hookrightarrow S$ is étale in codimension one, by tensoring $\phi_{i}^{(e)}$ with $E_{S}$ over $R$, we have the commutative diagram

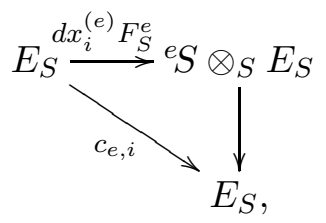

where $F_{S}^{e}: E_{S} \rightarrow{ }^{e} S \otimes_{S} E_{S}$ is the induced $e$-times iterated Frobenius map on $E_{S}$ (see the proof of [W, Theorem 2.7]). Hence $\operatorname{Ker}\left(d x_{i}^{(e)} F_{S}^{e}\right) \subseteq\left(0: c_{e, i}\right)_{E_{S}}$ for every $0 \leq e \leq e_{1}$ and $1 \leq i \leq r_{e}$. Since $\sum_{e=0}^{e_{1}} \sum_{i=1}^{r_{e}} c_{e, i}=c$, we have $c \cdot \bigcap_{e=0}^{e_{1}} \bigcap_{i=1}^{r_{e}} \operatorname{Ker}\left(d x_{i}^{(e)} F_{S}^{e}\right)=0$. By condition $(*)$, this implies that $c \in \tau\left((\mathfrak{a} S)^{t}\right)$.

Now we will show the latter assertion assuming that $R \hookrightarrow S$ is flat. By the above argument, we already know that $\tau\left((\mathfrak{a} S)^{t}\right) \supseteq \tau\left(\mathfrak{a}^{t}\right) S$. Suppose that $c \in \tau\left((\mathfrak{a} S)^{t}\right)$. Since $S$ is a free $R$-module, we can choose a basis $s_{1}, \ldots, s_{k}$ for $S$ over $R$ and write $c=\sum_{j=1}^{k} c_{j} s_{j}$ for $c_{j} \in R$. For any ideal $I \subseteq R$ and any $z \in I^{* \mathfrak{a}^{t}}$, clearly $z \in(I S)^{*(\mathfrak{a} S)^{t}}$. By the definition of $\tau\left((\mathfrak{a} S)^{t}\right)$, we have $\sum_{j=1}^{k} c_{j} z s_{j}=c z \in I S=\bigoplus_{j=1}^{k} I s_{j}$. It follows that $c_{j} z \in I$, therefore $c_{j} \in \tau\left(\mathfrak{a}^{t}\right)$ for every $j=1, \ldots, k$. Thus $c \in \tau\left(\mathfrak{a}^{t}\right) S$.

The following example shows that the last equality in Theorem 3.3 breaks down in the absence of the flatness, even for the case $\mathfrak{a}=R$.

ExAmple 3.4. Let $S=k[[x, y, z]] /\left(x^{n}+y^{n}+z^{n}\right)$ be the Fermat hypersurface of degree $n$ over a field of characteristic $p \geq n+1, R=S^{(r)}$ the $r$ th Veronese subring of $S$, and assume that $n \geq 3$ and $r \geq 2$ are not divisible by $p$. Then $\tau(R)=R_{\geq\lfloor 1+(n-3) / r\rfloor}=R_{\geq\lceil(n-2) / r\rceil}$ (note that $\lfloor 1+(n-3) / r\rfloor=\lfloor(n-2) / r+(r-1) / r\rfloor=\lceil(n-2) / r\rceil)$ and $\tau(S)=S_{\geq n-2}$. Hence $\tau(S) \cap R=R_{\geq\lceil(n-2) / r\rceil}=\tau(R)$, but $\tau(R) S=S_{\geq\lceil(n-2) / r\rceil r} \subsetneq S_{\geq n-2}=$ $\tau(S)$ if $n-2$ is not divisible by $r$.

\section{§4. Lipman-Skoda's theorem}

In [Li], Lipman proves under the Grauert-Riemenschneider vanishing theorem that, if $\mathfrak{a}$ is an ideal of a regular local ring with a reduction generated by $l$ elements, then $\mathcal{J}\left(\mathfrak{a}^{l}\right)=\mathcal{J}\left(\mathfrak{a}^{l-1}\right) \mathfrak{a}$, where $\mathcal{J}(\mathfrak{b})$ denotes the multiplier ideal (or adjoint ideal in the sense of [Li]) associated to an ideal $\mathfrak{b}$. 
This result is called Skoda's theorem [La] and formulated algebraically by Lipman in his proof of "modified Briançon-Skoda theorem." We give a simple proof of the corresponding equality for the ideal $\tau\left(\mathfrak{a}^{l}\right)$. The following version is just a refinement of [HY, Theorem 2.1]; see also Remark 1.2 for the definition of the ideal $\tau\left(\mathfrak{a}^{l} \mathfrak{b}^{t}\right)$ with "bi-exponents."

THEOREM 4.1. Let $(R, \mathfrak{m})$ be a complete local ring of characteristic $p>0$ and let $\mathfrak{a} \subseteq R$ be an ideal of positive height with a reduction generated by $l$ elements. Let $\mathfrak{b}$ be an ideal of $R$ such that $\mathfrak{b} \cap R^{\circ} \neq \emptyset$ and $t \geq 0$ a rational number. Then

$$
\tau\left(\mathfrak{a}^{l} \mathfrak{b}^{t}\right)=\tau\left(\mathfrak{a}^{l-1} \mathfrak{b}^{t}\right) \mathfrak{a}
$$

Proof. We will see that

$$
0_{M}^{* \mathfrak{a}^{l} \mathfrak{b}^{t}}=0_{M}^{* \mathfrak{a}^{l-1} \mathfrak{b}^{t}}: \mathfrak{a} \text { in } M
$$

for any $R$-module $M$. By fundamental properties of $\mathfrak{a}^{t}$-tight closure [HY,

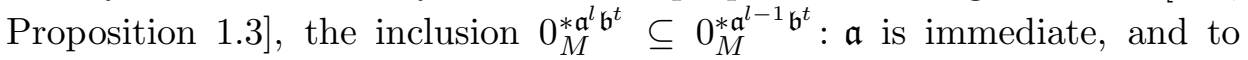
prove the reverse inclusion, we may assume without loss of generality that $\mathfrak{a}$ itself is generated by $l$ elements. Let $z \in 0_{M}^{* \mathfrak{a}^{l-1} \mathfrak{b}^{t}}: \mathfrak{a}$, i.e., $z \mathfrak{a} \subseteq 0_{M}^{* \mathfrak{a}^{l-1} \mathfrak{b}^{t}}$. Then there exists $c \in R^{\circ}$ such that $c z^{q} \mathfrak{a}^{[q]}\left(\mathfrak{a}^{l-1}\right)^{q} \mathfrak{b}^{\lceil t q\rceil}=0$ in $\mathbb{F}^{e}(M)$ for all $q=p^{e} \gg 0$. Since $\mathfrak{a}$ is generated by $l$ elements, one has $\mathfrak{a}^{q l}=\mathfrak{a}^{q(l-1)} \mathfrak{a}^{[q]}$, so that $c z^{q} \mathfrak{a}^{l q} \mathfrak{b}^{\lceil t q\rceil}=0$ for all $q=p^{e} \gg 0$, that is, $z \in 0_{M}^{* \mathfrak{a}^{l} \mathfrak{b}^{t}}$. Thus we have $0_{M}^{* \mathfrak{a}^{l} \mathfrak{b}^{t}}=0_{M}^{* \mathfrak{a}^{l-1} \mathfrak{b}^{t}}: \mathfrak{a}$.

Now assume that $(R, \mathfrak{m})$ is a complete local ring and let $E=E_{R}(R / \mathfrak{m})$, the injective envelope of the $R$-module $R / \mathfrak{m}$. Then by the Matlis duality, $\operatorname{Ann}_{E}\left(\tau\left(\mathfrak{a}^{l-1} \mathfrak{b}^{t}\right)\right)$ is equal to the union of $0_{M}^{* \mathfrak{a}^{l-1} \mathfrak{b}^{t}}$ taken over all finitely generated $R$-submodules $M$ of $E$. Hence, if $z \in \operatorname{Ann}_{E}\left(\tau\left(\mathfrak{a}^{l-1} \mathfrak{b}^{t}\right) \mathfrak{a}\right)$, then there exists a finitely generated submodule $M \subset E$ such that $z \in\left(0_{M}^{* \mathfrak{a}^{l-1} \mathfrak{b}^{t}}: \mathfrak{a}\right)_{E}$.

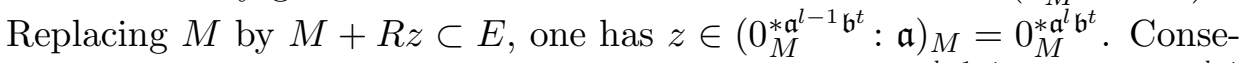
quently, $\operatorname{Ann}_{E}\left(\tau\left(\mathfrak{a}^{l-1} \mathfrak{b}^{t}\right) \mathfrak{a}\right)$ is equal to the union of $\left(0_{M}^{* \mathfrak{a}^{l-1} \mathfrak{b}^{t}}: \mathfrak{a}\right)_{M}=0_{M}^{* \mathfrak{a}^{l} \mathfrak{b}^{t}}$ taken over all finitely generated submodules $M \subset E$. Therefore

$$
\tau\left(\mathfrak{a}^{l} \mathfrak{b}^{t}\right)=\bigcap_{M \subset E} \operatorname{Ann}_{R}\left(0_{M}^{* \mathfrak{a}^{l} \mathfrak{b}^{t}}\right)=\operatorname{Ann}_{R}\left(\operatorname{Ann}_{E}\left(\tau\left(\mathfrak{a}^{l-1} \mathfrak{b}^{t}\right) \mathfrak{a}\right)\right)=\tau\left(\mathfrak{a}^{l-1} \mathfrak{b}^{t}\right) \mathfrak{a}
$$

The characterization of the ideal $\tau\left(\mathfrak{a}^{t}\right)$ given in Lemma 2.1 enables us to replace the completeness assumption in the above theorem by condition $(*)$. 
TheOREM 4.2. Let $(R, \mathfrak{m})$ be an F-finite local ring of characteristic $p>0$ and let $\mathfrak{a} \subseteq R$ be an ideal of positive height with a reduction generated by $l$ elements. Let $\mathfrak{b}$ be an ideal of $R$ such that $\mathfrak{b} \cap R^{\circ} \neq \emptyset$ and $t$ a nonnegative rational number. If condition (*) is satisfied for $\mathfrak{a}^{l-1} \mathfrak{b}^{t}$ and $\mathfrak{a}^{l} \mathfrak{b}^{t}$, then

$$
\tau\left(\mathfrak{a}^{l} \mathfrak{b}^{t}\right)=\tau\left(\mathfrak{a}^{l-1} \mathfrak{b}^{t}\right) \mathfrak{a}
$$

Proof. We may assume without loss of generality that $\mathfrak{a}$ is generated by $l$ elements, so that $\mathfrak{a}^{q l}=\mathfrak{a}^{q(l-1)} \mathfrak{a}^{[q]}$ for all $q=p^{e}$. We fix an element $d \in R^{\circ}$ such that $R_{d}$ is regular. Then, thanks to Remark 2.5, some power $d^{n}$ is an $\mathfrak{a}^{k} \mathfrak{b}^{t}$-test element for all $k \geq 0$.

By Lemma 2.1, an element $c \in R$ is in $\tau\left(\mathfrak{a}^{l} \mathfrak{b}^{t}\right)$ if and only if there exist finitely many $R$-homomorphisms $\phi_{i}^{(e)} \in \operatorname{Hom}_{R}\left(R^{1 / p^{e}}, R\right)$ for $0 \leq e \leq e_{1}$ and $1 \leq i \leq r_{e}$ such that

$$
c \in \sum_{e=0}^{e_{1}} \sum_{i=1}^{r_{e}} \phi_{i}^{(e)}\left(\left(d^{n} \mathfrak{a}^{p^{e} l} \mathfrak{b}^{\left\lceil t p^{e}\right\rceil}\right)^{1 / p^{e}}\right) .
$$

Since

$$
\begin{aligned}
\phi_{i}^{(e)}\left(\left(d^{n} \mathfrak{a}^{p^{e} l} \mathfrak{b}^{\left\lceil t p^{e}\right\rceil}\right)^{1 / p^{e}}\right) & =\phi_{i}^{(e)}\left(\left(d^{n} \mathfrak{a}^{p^{e}(l-1)} \mathfrak{a}^{\left[p^{e}\right]} \mathfrak{b}^{\left\lceil t p^{e}\right\rceil}\right)^{1 / p^{e}}\right) \\
& =\phi_{i}^{(e)}\left(\left(d^{n} \mathfrak{a}^{p^{e}(l-1)} \mathfrak{b}^{\left\lceil t p^{e}\right\rceil}\right)^{1 / p^{e}}\right) \mathfrak{a} \\
& \subseteq \tau\left(\mathfrak{a}^{l-1} \mathfrak{b}^{t}\right) \mathfrak{a}
\end{aligned}
$$

again by Lemma 2.1, this is equivalent to saying that $c \in \tau\left(\mathfrak{a}^{l-1} \mathfrak{b}^{t}\right) \mathfrak{a}$.

Corollary 4.3. (Modified Briançon-Skoda, cf. [BSk], [HY], [HH2], [Li]) Let $(R, \mathfrak{m})$ and $\mathfrak{a} \subseteq R$ be as in Theorem 4.1 or 4.2 . Then

$$
\tau\left(\mathfrak{a}^{n+l-1}\right) \subseteq \mathfrak{a}^{n}
$$

for all $n \geq 0$. In particular, if $R$ is weakly F-regular, then $\overline{\mathfrak{a}^{n+l-1}} \subseteq \mathfrak{a}^{n}$ for all $n \geq 0$.

Corollary 4.4. Let $(R, \mathfrak{m})$ be a d-dimensional local ring of characteristic $p>0$ with infinite residue field $R / \mathfrak{m}$ and $\mathfrak{a} \subseteq R$ an ideal of positive height. Let $\mathfrak{b}$ be an ideal of $R$ such that $\mathfrak{b} \cap R^{\circ} \neq \emptyset$ and $t$ a nonnegative rational number. We assume that $(R, \mathfrak{m})$ is complete or it is F-finite and 
condition $(*)$ is satisfied for $\mathfrak{a}^{n+d-1} \mathfrak{b}^{t}$ for every $n \geq 0$. Then for any $n \geq 0$ one has

$$
\tau\left(\mathfrak{a}^{n+d-1} \mathfrak{b}^{t}\right)=\tau\left(\mathfrak{a}^{d-1} \mathfrak{b}^{t}\right) \mathfrak{a}^{n}
$$

Proof. We can assume that $\mathfrak{a}$ is a proper ideal of $R$. Since the residue field $R / \mathfrak{m}$ is infinite, by [NR], $\mathfrak{a}$ has a reduction ideal generated by at most $d$ elements. Therefore the assertion immediately follows from Theorems 4.1 and 4.2 .

EXAMPLE 4.5. (cf. [HY, Theorem 2.15]) Let $R$ be a $d$-dimensional regular local ring of characteristic $p>0$ with the maximal ideal $\mathfrak{m}$. Then

$$
\tau\left(\mathfrak{m}^{n}\right)= \begin{cases}R & \text { if } n<d \\ \mathfrak{m}^{n-d+1} & \text { if } n \geq d\end{cases}
$$

In particular, $\tau\left(\mathfrak{m}^{d-1}\right) \supsetneq \tau\left(\mathfrak{m}^{d-2}\right) \mathfrak{m}$. This shows that $l \geq d$ is the best possible bound for the equality $\tau\left(\mathfrak{m}^{l}\right)=\tau\left(\mathfrak{m}^{l-1}\right) \mathfrak{m}$ in this case.

Remark 4.6. Let $(R, \mathfrak{m})$ and $\mathfrak{a}$ be as in Theorem 4.1 or 4.2 and let $\mathfrak{q}$ be any reduction of $\mathfrak{a}$. Then $\tau\left(\mathfrak{a}^{l-1}\right) \mathfrak{a}=\tau\left(\mathfrak{a}^{l-1}\right) \mathfrak{q}$. In particular, $\tau\left(\mathfrak{a}^{l-1}\right)=$ $\tau\left(\mathfrak{q}^{l-1}\right)$ is contained in the coefficient ideal $\mathfrak{c}(\mathfrak{a}, \mathfrak{q})$ of $\mathfrak{a}$ with respect to $\mathfrak{q}$; $\mathrm{cf}$. $[\mathrm{AH}]$.

If $R$ is Gorenstein, $\mathfrak{a}$ is $\mathfrak{m}$-primary with minimal reduction $\mathfrak{q}$ and if the Rees algebra $R[\mathfrak{a} t]$ is F-rational, then the equality $\tau\left(\mathfrak{a}^{d-1}\right)=\mathfrak{c}(\mathfrak{a}, \mathfrak{q})$ holds; see $[\mathrm{HY}]$ and Hyry [Hy].

\section{REFERENCES}

[AH] I. Aberbach and C. Huneke, An improved Briançon-Skoda theorem with applications to the Cohen-Macaulayness of Rees algebras, Math. Ann., 297 (1993), 67-114.

[AM] I. Aberbach and B. MacCrimmon, Some results on test elements, Proc. Edinburgh Math. Soc., (2) 42 (1999), 541-549.

[BSm] A. Bravo and K.E. Smith, Behavior of test ideals under smooth and étale homomorphisms, J. Algebra, 247 (2002), 78-94.

[BSk] J. Briançon and H. Skoda, Sur la clôture intégrale dún idéal de germes de fonctions holomorphes en un point de $C^{n}$, C. R. Acad. Sci. Paris Sér. A, 278 (1974), 949-951. 
[DEL] J.-P. Demailly, L. Ein and R. Lazarsfeld, A subadditivity property of multiplier ideals, Michigan Math. J., 48 (2000), 137-156.

[HW] N. Hara and K.-i. Watanabe, F-regular and F-pure rings vs. log terminal and log canonical singularities, J. Algebraic Geom., 11 (2002), 363-392.

[HY] N. Hara and K. Yoshida, A generalization of tight closure and multiplier ideals, Trans. Amer. Math. Soc., 355 (2003), 3143-3174.

[HH1] M. Hochster and C. Huneke, Tight Closure and strong F-regularity, Mem. Soc. Math. France, 38 (1989), 119-133.

[HH2] Tight closure, invariant theory and the Briançon-Skoda theorem, J. Amer. Math. Soc., 3 (1990), 31-116.

[HH3] - F-regularity, test elements, and smooth base change, Trans. Amer. Math. Soc., 346 (1994), 1-62.

[Hy] E. Hyry, Coefficient ideals and the Cohen-Macaulay property of Rees algebras, Proc. Amer. Math. Soc., 129 (2001), 1299-1308.

[La] R. Lazarsfeld, Positivity in algebraic geometry, book, to appear.

[Li] J. Lipman, Adjoints of ideals in regular local rings, Math. Research Letters, 1 (1994), 739-755.

[LS1] G. Lyubeznik and K.E. Smith paper Strong and weak F-regularity are equivalent for graded rings, Amer. J. Math., 121 (1999), 1279-1290.

[LS2] - On the commutation of the test ideal under localization and completion, Trans. Amer. Math. Soc., 353 (2001), no. 8, 3149-3180.

[NR] D.G. Northcott and D. Rees, Reductions of ideals in local rings, Proc. Camb. Philos. Soc., 50 (1954), 145-158.

[Ta] S. Takagi, An interpretation of multiplier ideals via tight closure, J. Algebraic Geom., 13 (2004), 393-415.

[W] K.-i. Watanabe, F-regular and F-pure normal graded rings, J. Pure Appl. Algebra, 71 (1991), 341-350.

Nobuo Hara

Mathematical Institute

Tohoku University

Sendai 980-8578

Japan

hara@math.tohoku.ac.jp 
Shunsuke Takagi

Graduate School of Mathematical Sciences

University of Tokyo

3-8-1, Komaba, Meguro Tokyo 153-8914 Japan

stakagi@ms.u-tokyo.ac.jp

CURRENT ADDRESS:

Faculty of Mathematics

Kyushu University

6-10-1, Hakozaki, Higashi-ku

Fukooka 812-8581

Japan

stagagi@math.kyushu-u.ac.jp 